\title{
Cross-pathway Control of Ornithine Carbamoyltransferase Synthesis in Neurospora crassa
}

\author{
By BARBARA F. KEMP† AND HARRY J. FLINT* \\ Department of Genetics, University of Nottingham, University Park, Nottingham NG7 2RD, \\ U.K.
}

(Received 10 August 1981; revised 26 October 1981)

The pattern of cross-pathway regulation of the arginine synthetic enzyme ornithine carbamoyltransferase was investigated in Neurospora crassa, using single and double mutant auxotrophic strains starved for their required amino acids. These experiments show that starvation for histidine, tryptophan, isoleucine, valine or arginine can result in derepression of ornithine carbamoyltransferase. Methionine starvation also gave slight derepression, but starvation for lysine or leucine gave little or no effect.

\section{INTRODUCTION}

Ornithine carbamoyltransferase (OCTase), in common with several other enzymes of arginine biosynthesis, has been shown to increase in activity in response to low levels of arginine, tryptophan or histidine in Neurospora crassa mycelium. Similar 'cross-pathway' regulation is also shown by enzymes involved in the tryptophan, histidine and lysine pathways (Carsiotis et al., 1974; Carsiotis \& Jones, 1974; Flint \& Kemp, 1981; Spurgeon \& Matchett, 1977). We have investigated the 'spectrum' of amino acids capable of eliciting derepression of OCTase, by examining the ability of various auxotrophic strains to derepress the enzyme when starved of their required amino acid. The use of single mutant strains is complicated by possible differences in genetic background between strains, which might influence the derepression observed. The main approach used was to construct a series of double mutant strains, carrying a common arginine auxotrophic marker together with a second amino acid requirement. This allows direct comparison of the response to arginine deprivation and to the deprivation of a second amino acid within a single strain.

\section{METHODS}

Strains. The following strains carrying single auxotrophic requirements were obtained from the Fungal Genetics Stock Center, Humbolt State University Foundation, Arcata, Calif., U.S.A.: trp-3A (FGSC 862), his-2A (FGSC 950), lys-2A (FGSC 2163), ilv-1A (FGSC 500), leu-1A (FGSC 28), met-1A (FGSC 3049) and arg-5a (FGSC 480). The St Lawrence wild-type strain was obtained originally from the stock collection of $\mathrm{H}$. Kacser. Double mutant combinations were obtained by isolating and testing single ascospores derived from crosses between $\arg 5 a$ and each of the other mutant strains made using $1.5 \%(\mathrm{w} / \mathrm{v})$ cornmeal agar supplemented with $0.05 \%$ sucrose and required amino acids at $0.005 \%$. All double mutant isolates used were of the same mating type (A).

Growth and starvation conditions. Mycelium was grown at $29^{\circ} \mathrm{C}$ in 1 litre conical flasks in a Gallenkamp orbital shaker, at $200 \mathrm{rev} . \mathrm{min}^{-1}$. Each flask contained $200 \mathrm{ml}$ Vogel's minimal medium (Vogel, 1956) with $4.5 \mathrm{~g}$ glucose and appropriate amino acid supplementation, and was inoculated with about $10^{7}$ conidia. Starvation of

$\dagger$ Present address: Department of Genetics, University of Leeds, Leeds LS2 9JT, U.K.

$\ddagger$ Present address: Department of Biology, Cave Hill Campus, University of the West Indies, Barbados, West Indies. 
single mutant strains was achieved by providing them with low initial concentrations of their required amino acid (normally 0.06 or $0.03 \mathrm{~mm}$ ) and allowing $43 \mathrm{~h}$ for the exhaustion of this supply. Double mutants were allowed to grow to between 0.2 and $0.5 \mathrm{mg}$ dry weight $\mathrm{ml}^{-1}$ on medium containing all their required amino acids (at concentrations of $1.2 \mathrm{~mm}$ ). The mycelium was then harvested by filtration, rinsed in $50 \mathrm{ml}$ of transfer medium and returned to medium lacking one or all of the required amino acids. Flasks were harvested at 8 and $23 \mathrm{~h}$ after transfer, and the mycelial pads rapidly frozen and lyophilized.

Ornithine carbamoyltransferase activity. OCTase activity was determined on extracts of powdered, lyophilized mycelium as described previously (Flint \& Kemp, 1981), except that assays were routinely performed on highly diluted crude extracts (final protein concentration in reaction mix about $0.01 \mathrm{mg} \mathrm{ml}^{-1}$ ) without dialysis. This procedure had been found to give values very similar to those obtained after dialysis, for mycelia grown under a variety of conditions. All assays were performed in duplicate.

\section{RESULTS AND DISCUSSION}

The effect of amino acid starvation upon OCTase activity was investigated in six double auxotrophic strains (Fig. 1). All markers used had previously been shown to be substantially 'non-leaky', and in all cases the omission of one or more of the required amino acids from the transfer medium caused a progressive reduction in growth by comparison with cultures transferred on to fully supplemented medium (results not shown), confirming that starvation conditions had been established. The results show that histidine, tryptophan, isoleucine and valine, in addition to arginine, are capable of eliciting the derepression of OCTase. In contrast, lysine and leucine were comparatively ineffective in causing derepression. This difference cannot easily be attributed to strain variation, as the arg-5 lys-2 and arg-5 leu-1 strains both gave appreciable derepression when starved for arginine.

The arg-5 met-1 strain showed low derepression for both arginine and methionine starvation. This may reflect a difference in general control properties between this and the other strains. On the other hand, the variation observed between different strains in their response to arginine starvation could be due to the presence of different amino acids in the medium prior to, and during, the exhaustion of arginine. In a series of 'reconstruction' experiments the $\arg -5$ single mutant strain was grown on each of the media employed for the growth of the double mutants in Fig. 1, and then transferred to medium lacking arginine but still containing the second amino acid. The pattern of OCTase derepression was found to be similar to that observed in response to arginine starvation of the double mutants, with the lowest derepression occurring in the presence of methionine $(2 \cdot 5$-fold $)$ and the highest in the presence of lysine or isoleucine plus valine (5- to 6-fold). The presence of methionine, in particular, in the medium of strains undergoing arginine starvation therefore appears to reduce the degree of OCTase derepression. The reason for this effect is not known.

Transfer of double mutant strains to minimal medium did not cause any synergistic effect, the resulting OCTase values being generally intermediate between those observed when amino acids were omitted singly (Fig. 1). However, it is unlikely that both of the required amino acids reach concentrations low enough to cause derepression after transfer to minimal medium. Once the concentration of one amino acid had fallen sufficiently to cause derepression and reduced overall protein synthesis, the requirement for the second amino acid would be substantially reduced, and its concentration would change little thereafter. It is still possible that a synergistic effect would be observed if the concentrations of two amino acids were simultaneously within the derepression range, but this situation is unlikely to be achieved in the experiments reported here.

Starvation of the parental single auxotrophic mutant strains for their required amino acid gave a pattern of derepression consistent with that observed in the double auxotrophs (Table 1). The derepression factors observed for arginine, tryptophan and histidine starvation were all higher than those observed in the double mutant strains, but this is almost certainly a consequence of the different starvation methods employed (see Methods), since from Fig. 1 OCTase activities can be seen to be still increasing in most cases, $23 \mathrm{~h}$ after transfer of the 


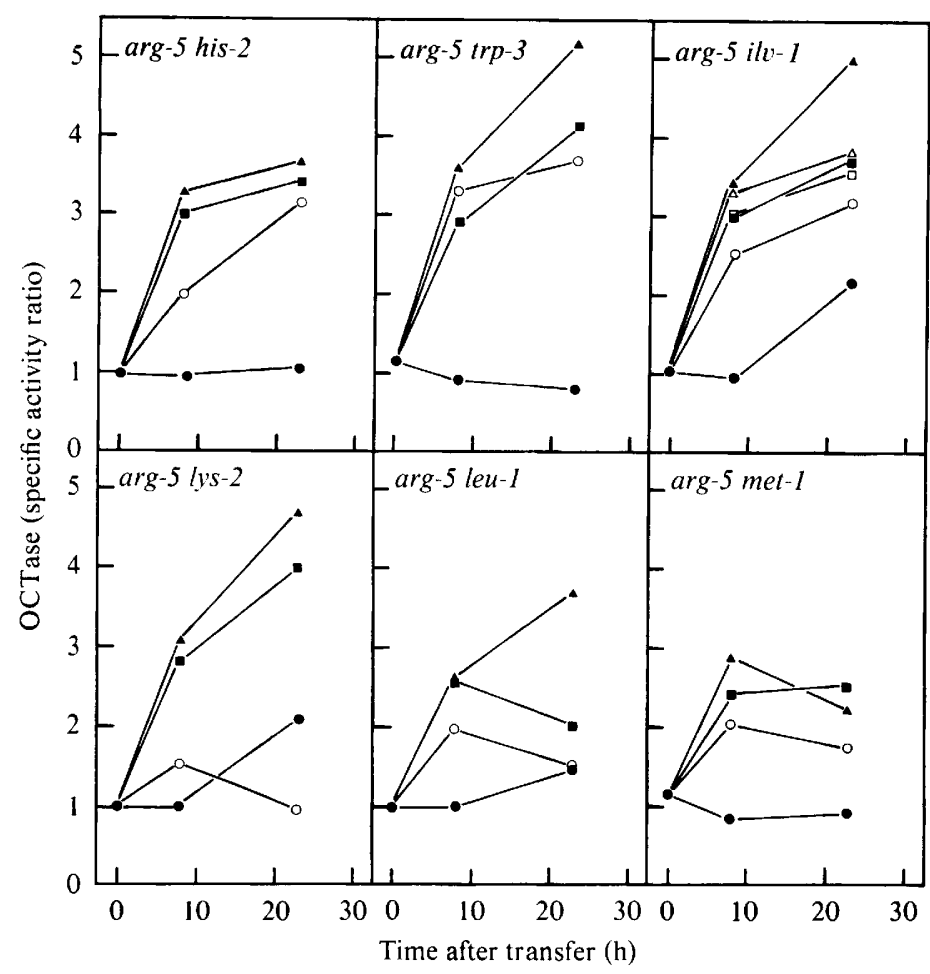

Fig. 1. Ornithine carbamoyltransferase activity in double auxotrophic mutant strains. Each strain was grown on medium containing all amino acids required for growth, and then transferred on to minimal medium ( $\square$ ), arginine-supplemented medium (O), medium lacking arginine but supplemented with the other required amino acid(s) (A) or medium containing all required amino acids (O). The arg-5 ilv-1 strain was also transferred on to medium supplemented with arginine plus isoleucine $(\triangle)$ or arginine plus valine. ( $\square$ ). All amino acids were present at concentrations of $1.2 \mathrm{mM}$. The specific activity ratio for OCTase is the activity relative to the basal level for each strain, taken as the mean of the 0 and $8 \mathrm{~h}$ time points for growth on medium containing all required amino acids. Points represent single harvests. The actual basal activities were $\left[\mu \mathrm{mol}(\mathrm{mg} \text { protein })^{-1} \mathrm{~min}^{-1}\right]:$ arg-5 his -20.61 , arg-5 trp-3 0.36 , arg-5 ilv-1 0.39 , arg-5 lys-2 0.79, arg-5 leu-1 0.79, arg-5 met-1 0.59.

double mutants, whereas the values for the single mutants represent maximal levels. In contrast, the values obtained for lysine and leucine starvation were lower in the single mutants, with the lys-2 strain showing apparent repression of OCTase as a consequence of starvation. In these two cases OCTase activity appeared to be decreasing after $23 \mathrm{~h}$ in the corresponding treatments with the double mutant strains, following an initial period of slight derepression (Fig. 1).

Appreciable (2- to 3-fold) variation in basal OCTase levels was observed both among the single mutant strains (Table 1) and among the double mutants (see legend to Fig. 1) during growth on fully supplemented media. This again appears to be largely a consequence of supplementation with different amino acids, since a wild-type strain grown on a range of amino acid supplemented media showed a similar pattern of variation in OCTase activity (Table 1). It is known that the addition of certain amino acids to the medium can cause substantial changes in the mycelial levels of other amino acids (unpublished results). Increases in amino acids above their minimal-grown levels do not appear to cause appreciable repression of OCTase in the wild-type strain (Flint \& Kemp, 1981). However, since the activities for mycelium grown on amino acid supplemented media were generally higher than for the minimal-grown wild type, the variation in 'basal' activity may be due to slight derepression resulting from reductions in certain amino acid pools. It is possible that the 


\section{Table 1. Effect of amino acid supplementation upon OCTase activity in wild-type and single auxotrophic mutant strains}

Amino acids were present at $1.2 \mathrm{~mm}$ in the non-starved condition, for which values represent means of at least two harvests taken between 0.3 and $1.0 \mathrm{mg}$ dry weight $\mathrm{ml}^{-1}$, when growth was exponential. Values for starved mycelia were obtained from cultures harvested $43 \mathrm{~h}$ after inoculation on to media containing 0.06 and $0.03 \mathrm{~mm}$ concentrations of the required amino acid. The value given represents the mean of the values for the two media, which differed from the mean by less than $10 \%$.

\begin{tabular}{|c|c|c|c|c|}
\hline \multirow{2}{*}{$\begin{array}{l}\text { Amino acid } \\
\text { supplement }\end{array}$} & \multirow[b]{2}{*}{ Strain } & \multicolumn{2}{|c|}{$\mu \mathrm{mol}(\mathrm{mg} \text { protein })^{-1} \mathrm{~min}^{-1}$} & \multirow{2}{*}{$\begin{array}{c}\text { Ratio } \\
\text { starved// } \\
\text { non-starved }\end{array}$} \\
\hline & & Non-starved & Starved & \\
\hline Arginine & $\begin{array}{l}\arg -5 \\
\text { WT }\end{array}$ & $\begin{array}{l}0.44 \\
0.58\end{array}$ & $2 \cdot 64$ & $6 \cdot 0$ \\
\hline Histidine & $\begin{array}{l}\text { his-2 } \\
\text { WT }\end{array}$ & $\begin{array}{l}0.48 \\
0.55\end{array}$ & $2 \cdot 71$ & $5 \cdot 7$ \\
\hline Tryptophan & $\begin{array}{l}\text { trp-3 } \\
\text { WT }\end{array}$ & $\begin{array}{l}0.39 \\
0.50\end{array}$ & $2 \cdot 06$ & $5 \cdot 3$ \\
\hline Isoleucine and valine & $\begin{array}{l}i l v-1 \\
\text { WT }\end{array}$ & $\begin{array}{l}0.55 \\
0.35\end{array}$ & $1 \cdot 54$ & $2 \cdot 8$ \\
\hline Lysine & $\begin{array}{l}\text { lys-2 } \\
\text { WT }\end{array}$ & $\begin{array}{l}0.73 \\
0.63\end{array}$ & 0.23 & $0 \cdot 3$ \\
\hline Leucine & $\begin{array}{l}\text { leu-l } \\
\mathrm{WT}\end{array}$ & $\begin{array}{l}0.96 \\
1.09\end{array}$ & 0.94 & 1.0 \\
\hline Methionine & $\begin{array}{l}\text { met-1 } \\
\text { WT }\end{array}$ & $\begin{array}{l}0.67 \\
0.70\end{array}$ & $1 \cdot 54$ & $2 \cdot 3$ \\
\hline None & WT & 0.39 & & \\
\hline
\end{tabular}

repression observed in the lys-2 strain during starvation (Table 1) in fact represents a return to the fully repressed state as other amino acids become restored to their repressive levels, lysine itself having no direct effect.

The deprivation of arginine, tryptophan, histidine, isoleucine or valine can lead to derepression of OCTase in Neurospora crassa mycelium. Methionine starvation also results in slight derepression, while lysine or leucine starvation has little or no effect. The spectrum of amino acids capable of eliciting OCTase derepression in $N$. crassa thus appears broadly similar to that found for the same enzyme in Aspergillus nidulans (Piotrowska, 1980), where arginine, histidine, tryptophan, or proline limitation is effective, while in yeast limitation of histidine, lysine, isoleucine or valine, in addition to arginine, have been shown to be effective (Delforge et al., 1975; Messenguy, 1979). The fact that not all amino acids are capable of eliciting cross-pathway derepression in these organisms suggests that the response is not triggered simply by a reduction in the overall rate of protein synthesis, or in the general level of tRNA charging. The failure of lysine to elicit derepression of OCTase in $N$. crassa contrasts with the observation that the lysine synthetic enzyme saccharopine dehydrogenase is derepressed in response to arginine or histidine deprivation (Flint \& Kemp, 1981). Thus the spectrum of amino acid capable of eliciting derepression is not identical with the spectrum of pathways in which enzymes can be derepressed.

We would like to acknowledge the support of the Science Research Council, Grant no. GR/A 72544.

\section{REFERENCES}

Carsiotis, M. \& Jones, R. F. (1974). Cross-pathway regulation: tryptophan mediated control of histidine and arginine biosynthetic enzymes in Neurospora crassa. Journal of Bacteriology 119, 889-892.

Carsiotis, M., Jones, R. F. \& Wesseling, A. C.
(1974). Cross-pathway regulation: histidine mediated control of histidine, tryptophan and arginine biosynthetic enzymes in Neurospora crassa. Journal of Bacteriology 119, 893-898.

Delforge, J., Messenguy, F. \& Wiame, J. M. 
(1975). The regulation of arginine biosynthesis in Saccharomyces cerevisiae. The specificity of $\arg R^{-}$ mutations and the general control of amino acid biosynthesis. European Journal of Biochemistry 57, 231-239.

Flint, H. J. \& KEMP, B. F. (1981). General control of arginine biosynthetic enzymes in Neurospora crassa. Journal of General Microbiology 124, 129-140.

Messenguy, F. (1979). Concerted repression of the synthesis of the arginine biosynthetic enzymes by amino acids: a comparison between the regulatory mechanism controlling amino acid biosynthesis in bacteria and yeast. Molecular and General Genetics 169, 85-95.
PIOTROWSKA, M. (1980). Cross-pathway regulation of ornithine carbamoyltransferase synthesis in Aspergillus nidulans. Journal of General Microbiology 116, 335-339.

Spurgeon, S. L. \& Matchett, W. H. (1977). Inhibition of aminoacyl-transfer ribonucleic acid synthetases and the regulation of amino acid biosynthetic enzymes in Neurospora crassa. Journal of Bacteriology 129, 1303-1312.

VoGel, H. J. (1956). A convenient growth medium for Neurospora (medium N). Microbial Genetics Bulletin 13, 42-43. 